\title{
Unexpected thoracic endograft infection following a colorectal procedure
}

\author{
Rong-Hsin Yang, Tse-Hao Lee and Yum-Kung Chu* \\ Department of Nuclear Medicine, Taipei Veterans General Hospital, Taipei, Taiwan
}

\begin{abstract}
Infection of endovascular stent-grafts is a severe complication, but not often reported. We report herein a case of a 61-year-old man presented with pneumonia-like symptoms 24 days after receiving laparoscopic resection of stage 1 sigmoid cancer. Acute pneumonitis and cancer recurrence were excluded on Ga-67 citrate imaging. An infected endovascular graft of the aortic arch, which had been placed 4 months previously, was depicted scintigraphically. Due to multiple comorbidities, the patient underwent decortications via video-assisted thoracic surgery plus aggressive drainage. The symptoms settled and the patient was discharged on oral levofloxacin after intravenous treatment with ertapenem and teicoplanin for six weeks. This report fuels the belief that subclinical bacteremia associated with various procedures may incur bacterial seeding on the preexisting endovascular devices, and causes secondary prosthetic infection. In selected cases such as ours, prosthetic graft preservation may provide a definite treatment. Continued awareness of potential septic complications in patients treating with an endovascular grafting is needed.
\end{abstract}

\section{Introduction}

Is not often reported [1,2], and remains a major diagnostic and therapeutic challenge [3]. With the expansion of endovascular procedures and increasing use of endografts and stents, infection deserves special attention [4]. The incidence of aortic stent-graft infection is 0.5 to $1 \%$, and untreated stent-graft infection can result in generalized sepsis and death [5]. Previous studies report that endografts would be more prone to bacterial contamination because of their placement in the vascular lumen [4]. Other sources of infection include perioperative contamination, remote site infection, mechanical erosion [2] and blood stream septicemia [6]. Complete graft removal including the debridement of infectious perigraft tissue is the preferred treatment [1]. Conservative treatment should be the only option for selected patients with high surgical risk [4].

\section{Case report}

A 61-year-old man, who had a laparoscopic anterior resection for a stage 1 sigmoid adenocarcinoma (pT2N0M0) 24 days ago, presented initially with spiking fevers up to $40^{\circ} \mathrm{C}$ and productive cough in the last 2 days. He had several comorbidities including diabetes mellitus, hypertension, emphysema, coronary arterial disease and gastric ulcer. Four months previously he had also undergone an endovascular repair with aortic-bi-carotid bypass grafting for an aortic arch aneurysm (Figure 1A) and an uneventful postoperative course.

Contrast-enhanced CT (Figure 1B) showed perihilar infiltration, mild bilateral pleural effusion with partial atelectasis of the left lower lobe of the lung, small opacity of right middle lung field, and some fluid collection around the descending aorta post thoracic aortic stent insertion. No sign of endoleak or cancer recurrence. Microbiological cultures of blood yielded Klebsiella pneumoniae. Based on septic manifestations, the patient was treated with cefuroxime empirically for suspicion of Klebsiella pneumoniae pneumonia, and he experienced transient improvement.

Two weeks after, the patient returned to the hospital with persistent fever and cough. On examination, he was pyrexial at $38.3^{\circ} \mathrm{C}$ and $\mathrm{BP}$ $130 / 68 \mathrm{mmHg}$. Leukocytosis $\left(13400 / \mathrm{mm}^{3}\right.$ with $85 \%$ of neutrophils), C-reactive protein rising $(20.52 \mathrm{mg} / \mathrm{dl})$, anemia $(\mathrm{Hgb} 8.3 \mathrm{mg} / \mathrm{dl})$ and mild proteinuria were evident. Blood cultures and antigen study for influenza $A$ and influenza B were negative. Ga-67 scan (Figure 2A) performed for cancer surveillance was notable for pathologic activity from the ascending aorta to proximal descending aorta, corresponding to the stent site. Acute pneumonitis and cancer recurrence were scintigraphically excluded. Under the impression of endograft infection, intravenous ertapenem (1 gm/day) was commenced.

Due to the poor clinical state and imminent risk for catastrophic complications, the patient underwent decortication, drainage and distal stent reconstruction through video-assisted thoracoscopic surgery (VATS) subsequently. The procedure was uneventful, and the pyrexia settled within $24 \mathrm{~h}$ after the surgery. Klebsiella pneumoniae was grown from pus culture, but negative from blood. As guided by the microbiology cultures, he completed a six-week course of intravenous antibiotics with ertapenem and teicoplanin. The sepsis completely resolved and inflammatory markers gradually returned (Figure 2B). On postoperative day 47, he was discharged on long-term oral levofloxacin. The patient remained well 4 months after stent-graft preservation, as did the CT scan appearance (Figure 1D).

\section{Discussion}

Presentations of endograft infection can vary from nonspecific

Correspondence to: Dr. Yum-Kung Chu, Department of Nuclear Medicine, Taipei Veterans General Hospital No. 201, Shipai Road Sec.2, Beitou District, Taipei City, Taiwan 11217, Tel: 886-2-28757301; Fax: 886-2-28715849; E-mail: ykchu@vghtpe.gov.tw

Key words: colorectal procedure, endograft infection, Ga-67, graft preservation, Klebsiella pneumoniae

Received: March 08, 2016; Accepted: April 15, 2016; Published: April 20, 2016 
signs (35.5\%) to overt sepsis [4]. Constitutional symptoms reported are chills and fever, stent-related discomfort, leukocytosis, elevated inflammatory markers, weight loss, nausea, and anemia [7]. Most cases $(54.9 \%)$ of endograft infection occurred more than four months after the primary procedure [7]. Implicated species in endograft infections include Staphylococcus, Streptococcus, Propionibacterium and Enterobacter, with the former two being the most common in the complication [2]. Risk factors for endovascular graft infection include advanced age, immunodeficiency, diabetes mellitus, site of the endovascular procedure, previous pseudoaneurysms, presence of cancers and subsequent surgeries [2,4]. Perioperative contamination seems to be the source of early infection. Secondary infection can occur from hematogenous seeding, or mechanical erosion $[2,6]$. Having diabetes and known malignancy our patient is predisposed to asymptomatic bacteremia, which may incur endograft infection. He presented with vague chest symptoms and his radiographic findings were inconclusive. Thus, any localized complaint in a patient with a relevant history of endovascular grafting should raise suspicion of endograft infection.

There is some evidence that abdominal surgery increases the risk of endograft infection. Gawenda $\mathrm{M}$ et al. reported endograft infection following a right hemicolectomy for a sigmoid cancer (pT3N0M0), and speculated that bacteremia developed during the bowel resection might have led to a late infection [1]. A similar scenario was noted in our patient who had endograft infection 24 days after a laparoscopic resection of sigmoid cancer. Development of endograft infection has also been described in patient following appendectomy [5] or with renal stone [8]. It is possible that preceding procedures or a consequence of prior lesions can precipitate subclinical bacteremia and bacterial seeding. It is noteworthy that Klebsiella pneumoniae was yielded from blood and pus cultures of our patient. Klebsiella pneumoniae is abundant in the gut microbiota and an enterobacteriae relevant to the development of colorectal cancer $[9,10]$. Nevertheless, we have no convincing evidence to establish a causal association between colorectal procedure and becteremia or endograft infection.

The diagnosis of endograft infections remains a major challenge [3], and is usually based on nonspecific septic findings, supported by imaging and microbiological investigations of the perigraft accumulation. However patients are often treated without knowing the causative organism, because suitable specimens are not obtained or because antibiotics have been instituted before head [3]. Contrastenhanced computed tomography (CT) may show air or fluid collection in the vicinity of a vascular graft [11]. As illustrated in our case, to distinguish hematomas, seromas and post-operative reaction from infected changes may be difficult and may not always provide definitive evidence of infection by CT scan $[3,12]$. On the other hand,

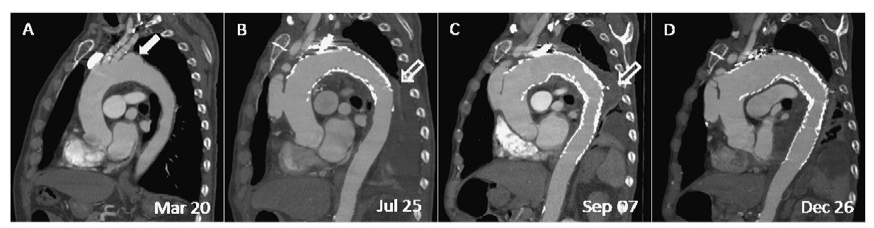

Figure 1. Serial computed tomography with sagittal reconstruction. (A) A saccular aneurysm was noted at the aortic arch (white arrow). (B) Sixteen weeks after endovascular aneurysm repair for the aneurysm, patient presented with fever and cough. CT depicted perihilar infiltration, bilateral pleural effusion and perigraft fluid collection (hollow arrow). (C) Loculated fluid collection with rim enhancement of proximal descending aorta (hollow arrow) was more appreciated, explaining as an inevitable reaction 2 weeks after distal stent reconstruction of the aorta. (D) A follow-up CT 4 months after stent-graft preservation and antibiotic treatment revealed clear improvement.

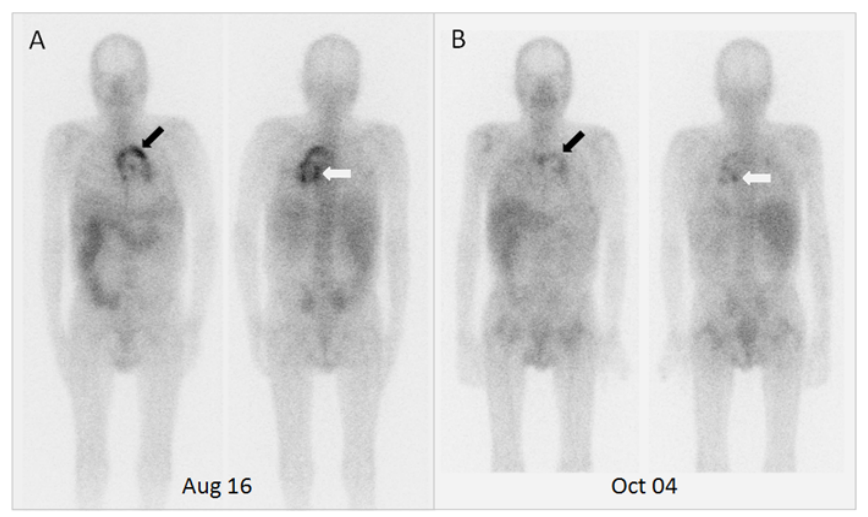

Figure 2. Ga-67 citrate whole body scanning. (A) Intense activity from the ascending aorta (black arrow) to proximal descending aorta (white arrow) in the vicinity of the prosthetic graft, suggesting graft infection. No active lesions were demonstrable in the lungs or abdomen. (B) Decortication plus intravenous antibiotics for 6 weeks resulted in remarkable improvement.

scintigraphic methods, such as Ga-67 citrate scan, F-18 FDG PET and labeled leukocytes study, are often used in screening of infectious disorders [13-15]. The importance of the radionuclide uptake pattern, namely focal uptake, has been pointed out for differential diagnosis of infectious and non-infectious conditions. In this case, Ga-67 citrate scan displayed the pathologic activity of the infected graft and excluded lung infections (Figure 2), better guiding for a diagnosis and further management.

Treatment of endograft infection follows the precepts of traditional surgical graft infections with removal of the endovascular material, debridement, and revascularization [1,4]. Specific antimicrobial treatment is a vital adjunct to surgical management [3]. Conservative treatment with abscess drainage and suppressive antibiotic therapy has been reported; however, it is not largely advocated as the best therapeutic approach [2]. Nevertheless, Calligaro et al. reported that complete or partial graft preservation combined with debridement and aggressive drainage is an acceptable option for treatment of entire endograft infection in selected patients with prohibitive risks for total graft excision [16]. These patients require long-term antibiotic treatment, namely, parenteral antibiotics for 6 weeks, followed by oral antibiotics for to 6 months [17]. Long-term imaging surveillance is also needed to ensure any disease progression is appropriately managed. More recently, prospective cohort studies found that in patients with infected stent-graft there was no difference in outcome with regard to vascular graft removal versus graft preservation $[18,19]$. In selected cases such as ours, prosthetic graft preservation may provide a definite treatment.

Prevention of graft infection is a vital concept and perioperative prophylactic strategies are of utmost importance [20]. Vascular surgeons should consider performing this type of endovascular procedure in the operating room environment, instead of the interventional radiology suite. Prophylactic antibiotics should also be given to prevent hematogenous bacterial seeding if the patient requires secondary revision or another unrelated surgery [1]. In fact, no consensus regarding the exact type and duration of prophylactic antibiotic use in this situation has been reached yet.

\section{Conclusion}

Endovascular graft infection can be challenging to diagnose and is associated with high mortality even if recognized and managed early. 
CT affords visualization of the structural changes, whereas radionuclide techniques allow such infections to be displayed on the basis of physiological changes. Management should follow the principles of any infected prosthesis adjunct with appropriate antibiotic coverage. This report highlights an unexpected association of endograft infection and an uneventful surgery of remote site. The continued awareness of potential septic complications in patients with a relevant history of endovascular grafting is needed.

\section{Conflict of interest statement}

On behalf of all authors, the corresponding author states that there is no conflict of interest.

\section{References}

1. Gawenda M, Aleksic M, Heckenkamp J, Krueger K, Brunkwall J (2004) Infections of Stent Grafts Following EVAR of AAA- An Underestimated Problem? EJVES Extra 8: $23-28$.

2. Ducasse E, Calisti A, Speziale F, Rizzo L, Misuraca M, et al. (2004) Aortoiliac stent graft infection: current problems and management. Ann Vasc Surg 18: 521-526. [Crossref]

3. FitzGerald SF, Kelly C, Humphreys H (2005) Diagnosis and treatment of prosthetic aortic graft infections: confusion and inconsistency in the absence of evidence or consensus. J Antimicrob Chemother 56: 996-999. [Crossref]

4. Lichtenfels E, Frankini AD, Cardozo MA, D'Azevedo PA (2011) Stent Graft Infection. $J$ Vasc Bras 10: 50-54.

5. Maleux G, Koolen M, Heye S (2009) Complications after endovascular aneurysm repair. Semin Intervent Radiol 26: 3-9. [Crossref]

6. Vogel TR, Symons R, Flum DR (2008) The incidence and factors associated with graft infection after aortic aneurysm repair. J Vasc Surg 47: 264-269. [Crossref]

7. Fiorani P, Speziale F, Calisti A, Misuraca M, Zaccagnini D, et al. (2003) Endovascular graft infection: preliminary results of an international enquiry. J Endovasc Ther 10: 919-927. [Crossref]

8. van den Berg HR, Leijdekkers VJ, Vahl A (2006) Aortic stent-graft infection following septic complications of a kidney stone. Cardiovasc Intervent Radiol 29: 443-445.

9. Wang T, Cai G, Qiu Y, Fei N, Zhang M, et al. (2012) Structural segregation of gut microbiota between colorectal cancer patients and healthy volunteers. ISME J 6: 320329. [Crossref]

10. 10. Huang WK, Chang JW, See LC, Tu HT, Chen JS, et al. (2012) Higher rate of colorectal cancer among patients with pyogenic liver abscess with Klebsiella pneumoniae than those without: an 11-year follow-up study. Colorectal Dis 14: e794801

11. Orton DF, LeVeen RF, Saigh JA, Culp WC, Fidler JL, et al. (2000) Aortic prosthetic graft infections: radiologic manifestations and implications for management. Radiographics 20: 977-993. [Crossref]

12. Fukuchi K, Ishida Y, Higashi M, Tsunekawa T, Ogino H, et al. (2005) Detection of aortic graft infection by fluorodeoxyglucose positron emission tomography: comparison with computed tomographic findings. J Vasc Surg 42: 919-25.

13. Bleeker-Rovers CP, van der Meer JW, Oyen WJ (2009) Fever of unknown origin. Semin Nucl Med 39: 81-87. [Crossref]

14. Love C, Palestro CJ (2013) Radionuclide imaging of inflammation and infection in the acute care setting. Semin Nucl Med 43: 102-113. [Crossref]

15. London K, Howman-Giles R (2008) Ga-67 and CT fusion imaging of an infected aortic graft. Clin Nucl Med 33: 41-43. [Crossref]

16. Calligaro KD, Veith FJ, Yuan JG, Gargiulo NJ, Dougherty MJ (2003) Intra-abdomina aortic graft infection: complete or partial graft preservation in patients at very high risk. J Vasc Surg 38: 1199-1205. [Crossref]

17. Bandyk DF (2002) Antibiotics-why so many and when should we use them? Semin Vasc Surg 15: 268-274. [Crossref]

18. Legout L, Sarraz-Bournet B, D’Elia PV, Devos P, Pasquet A, et al. (2012) Characteristics and prognosis in patients with prosthetic vascular graft infection: a prospective observational cohort study. Clin Microbiol Infect 18: 352-358. [Crossref]

19. Erb S, Sidler JA, Elzi L, Gurke L, Battegay M, et al. (2014) Surgical and antimicrobia treatment of prosthetic vascular graft infections at different surgical sites: a retrospective study of treatment outcomes. PLoS One 9: e112947.

20. Venkatesan AM, Kundu S, Sacks D, Wallace MJ, Wojak JC, et al. (2010) Practice guidelines for adult antibiotic prophylaxis during vascular and interventional radiology procedures. J Vasc Interv Radiol 21: 1611-1630.

Copyright: (C2016 Yang RH. This is an open-access article distributed under the terms of the Creative Commons Attribution License, which permits unrestricted use, distribution, and reproduction in any medium, provided the original author and source are credited. 